Journal of Computer Science 8 (1): 145-148, 2012

ISSN 1549-3636

(C) 2012 Science Publications

\title{
Performance Analysis on 16-Channels Wavelength Division Multiplexing in Free Space Optical Transmission under Tropical Regions Environment
}

\author{
Salasiah Hitam, Siti Norziela Suhaimi, Ahmad Shukri Mohd Noor, \\ Siti Barirah Ahmad Anas and Ratna Kalos Zakiah Sahbudin \\ Department of Computer and Communication System, \\ Faculty of Engineering, Univerisity Putra Malaysia, \\ 43400 UPM Serdang, Selangor, Malaysia
}

\begin{abstract}
Problem statement: Wavelength-Division-Multiplexing (WDM) is a promising technique for meeting the growing demand for increased bandwidth and various types of services in the optical access network. For wide area or metropolitan networks, fibers are deployed to provide huge bandwidth. In access networks, the fiber-to-the-home will partially solve the last mile problem. However, some environmentally sensitive area such as housing areas, tower buildings and national parks are not allowed to deploy fibers. Therefore, Radio Frequency (RF) is normally used to overcome this problem. The incompatibility of RF and optical channels is now widely believed to be the limiting factor in efforts to further increase transport capabilities. Free Space Optical (FSO) communication is the technology that can address any connectivity needed in optical networks, such as core, edge, or access networks. Approach: In this project, the simulation software namely Optical System version 7 is used to simulate the design of WDM in FSO transmission. The total losses that have been considered in this design are geometric loss, transmitter and receiver loss and atmospheric attenuation which focus on nonselective scattering during heavy rainfall condition in Malaysian environment. Malaysian weather data are used to reflect the conditions particularly in tropical regions. Results: We have presented the results of 16-channels WDM at $100-\mathrm{GHz}$ channel spacing. The simulated results show that this system can support a higher bit rate up to $2.5 \mathrm{Gbps}$ over $2.4 \mathrm{~km}$ distance. Conclusion: Simulation results showed that WDM FSO system may be a good candidate to solve the last mile problem and also it has capability to accommodate the channels more than 16. By introducing the error correction code or balance detection, the transmission distance might be increased further.
\end{abstract}

Key words: Free space optical, division multiplexing, selective scattering optical access, geometric loss, bit rate, old topic, receiver loss

\section{INTRODUCTION}

In a Wavelength Division Multiplexing (WDM)based access, the demand for bandwidth has increased in an extremely fast and is a promising solution for data transport in future all-optical wide area networks (AbRahman et al., 2009). At the same time, the cost of transporting information bit per $\mathrm{km}$ also needs to be reduced. WDM system in Free Space Optical (FSO) is the way to maximize the bandwidth usage but in low cost. It has been quite mature and applied to optical fiber networks universally. Several FSO WDM transmission system have been demonstrated successfully (Kintaka et al., 2010 and Sinefeld and Marom, 2010). Although FSO is an old topic, due to the availability of advanced technologies and high demand of broadband communications now, it becomes a new research area.

The advantages of using FSO are no license required from Federal Communications Commission (FCC) for FSO installation. Apart from that, the cost of installation is primarily economic because there is no extra cost of digging the street to lay fiber. In term of communication security, FSO uses narrow laser beam which makes detection, interception and jamming very difficult. Moreover, FSO hardware is also portable and quickly deployable (Cvijetic et al., 2010) WDM is a technology which multiplexes multiple optical carrier signals on a single medium by using different signals. It is the technique to carry many separate

Corresponding Author: Salasiah Hitam, Department of Computer and Communication System, Faculty of Engineering, University Putra Malaysia 43400 UPM Serdang, Selangor, Malaysia 
optical signals. Each signal modulated into different wavelength lasers and it transmitted at a different rate from the other signal.

In this study, the analysis on the performance of this system has been done under non-selective scattering in tropical environment. An optical wave propagating through the air experiences fluctuations in amplitude and phase due to atmospheric attenuation (Mie and Nonselective scattering), turbulence and scintillation.

\section{MATERIALS AND METHODS}

Atmospheric attenuation: In tropical country, rain dominates the total attenuation coefficient (Prokes, 2009). The attenuation due to rainfall is also called nonselective scattering since the drop size is much larger than the wavelengths making scattering effects wavelength independent (Singh, 2007). In this simulation, the data was taken from the Malaysian Meteorological Department. The atmospheric attenuation $\tau_{\mathrm{a}}$ is described by the following Beer's law Eq. 1 and 2 (Downing, 2004):

$\tau \alpha=\mathrm{e}-{ }^{(\text {(Babs }+\beta s c a t)} \mathrm{R}$

Where:

$$
\begin{aligned}
\beta_{\mathrm{abs}} \text { and } \beta_{\mathrm{scat}}= & \text { The absorption and scattering } \\
& \text { coefficient, respectively } \\
\mathrm{R} & =\text { The link distance }
\end{aligned}
$$

The total scattering by rain is given by (Achour, 2002):

$$
\beta_{\text {scat }}^{\text {rain }}=\sum_{\alpha} \pi \alpha^{2} N \alpha Q_{\text {scat }}\left(\frac{\alpha}{\lambda}\right)
$$

where, $Q_{\text {scat }}$ is the scattering efficiency, also referred to as the Mie attenuation coefficient. For ratios $a / \lambda>30$ the $\mathrm{Q}_{\text {scat }}$ is approximated as equal to $2 . \mathrm{N}_{\mathrm{a}}$ is the number of dropsof radius a in a unit volume $\left(\mathrm{cm}^{3}\right)$. Fig. 1 below shows atmospheric attenuation versus distance, throughout the year of 2000. It is linearly proportional with $5 \mathrm{~dB} \mathrm{~km}^{-1}$.

System design: In this project, the simulator software namely Optical System (Optisys) version 7 is used to simulate the design of WDM in FSO transmission. The block diagram of system design is shown in Fig. 2.

From Fig. 2, the transmitter part consists of 16 laser arrays with $10 \mathrm{dBm}$ input power each. The wavelength ranging for the input laser is from $1550 \mathrm{~nm}$ to $1552 \mathrm{~nm}$ with $100 \mathrm{GHz}$ interspacing between channels. The signals are transmitted through free space as a transmission channel with $8.86 \mathrm{~dB} \mathrm{~km}^{-1}$ attenuation for the worst case scenario (Chan, 2006).

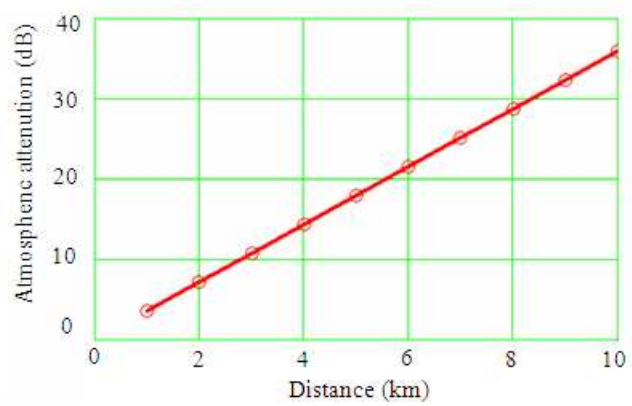

Fig. 1: Atmospheric attenuation as a function of link distance

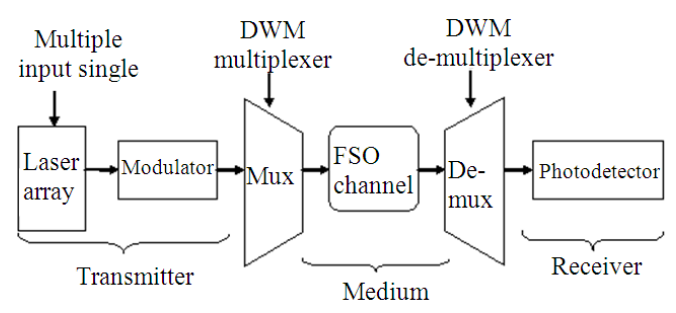

Fig. 2: Block diagram of WDM system in FSO transmission

Table 1: Typical parameters used in the simulation for the signal model and the noise model

\begin{tabular}{ll}
\hline & Signal model \\
\hline Transmitter power & $\mathrm{P}_{\mathrm{T}}=10 \mathrm{dBm}$ \\
Optical loss & $\mathrm{L}_{\mathrm{OP}}=9 \mathrm{~dB}$ \\
Atmospheric loss (heavy rainfall) & $8.86 \mathrm{~dB}$ \\
Pointing loss & $\mathrm{L}_{\mathrm{MP}}=3 \mathrm{~dB}$ \\
Quantum efficiency & $\eta=0.8$ \\
Operating wavelength & $\lambda=1550 \mathrm{~nm}$ \\
Planck's constant & $\mathrm{h}=6.66 \times 10^{-34} \mathrm{Js}$ \\
Distance & $\mathrm{R}=1-10 \mathrm{~km}$ \\
Speed of light & $\mathrm{c}=3 \times 10^{8} \mathrm{~m} \mathrm{sec}^{-1}$ \\
Noise model & \\
Bolzmann's constant & $\mathrm{k}=1.38 \times 10^{-23} \mathrm{~J} / \mathrm{K}$ \\
Absolute temperature & $\mathrm{T}=290 \mathrm{~K}$ \\
Observation bandwidth & $\mathrm{B}=311 \mathrm{MHz}$ \\
Dark current & $\mathrm{I}_{\mathrm{DC}}=100 \mu \mathrm{A}$ \\
\hline
\end{tabular}

This system needs the optical amplifiers for every channel to amplify the signals due to FSO losses after the photodetector. For simulation purposes, the parameters as listed in Table 1 are used which they are selected by referring to the actual values in the market and commercial product (Kim et al., 1998).

Design parameter: Any changes to design parameters will also change the performance of the circuit. Two most significant design parameters considered are the distance and bit rate.

Performance parameter: The performance parameters that will be observed are the Bit Error-Rate (BER) and 
output power To make a good optical receiver design, it is critical to understand and varying the different parameters that will affect the overall receiver performance.

\section{RESULTS}

Figure 3 shows the effect of distance to the system parameter. Bit rate of $2.5 \mathrm{Gbps}$ was used in this simulation as it was the highest bit rate used in the commercial product (LWPS, 2009). The power transmitting was set to $10 \mathrm{dBm}$ and normally the range of input power is from $0-13 \mathrm{dBm}$ for FSO (AC, 2011).

Figure 4 shows the effect of Bit Rate to the system performance. The distance is set to $2.4 \mathrm{~km}$ and the power transmitting is $10 \mathrm{dBm}$. Bit rates ranging from $0.155-10$ Gbps is used during this simulation. Number of channel set to 16 channels and the spacing between channels is set to $100 \mathrm{GHz}(0.8 \mathrm{~nm})$ based on ITU standard. The wavelength used in this simulation is $1550 \mathrm{~nm}$.

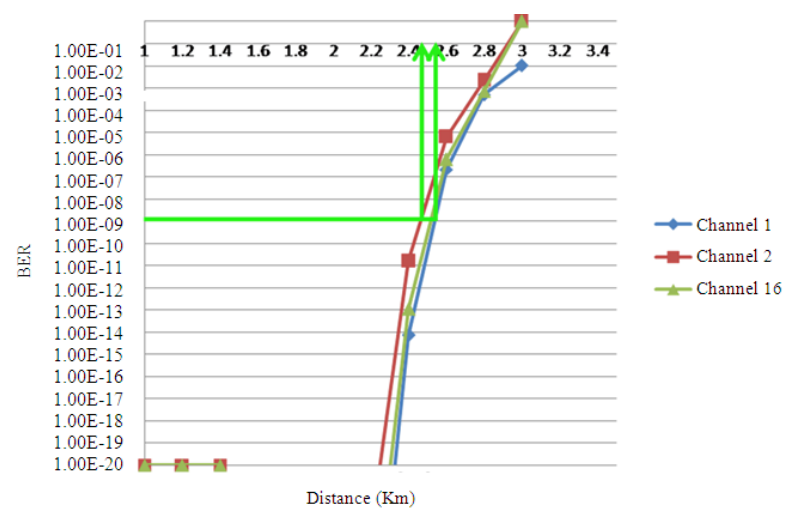

Fig. 3: BER in Relation to Increasing distance for WDM FSO system operating at $2.5 \mathrm{Gbps}$ under the condition of heavy rainfall

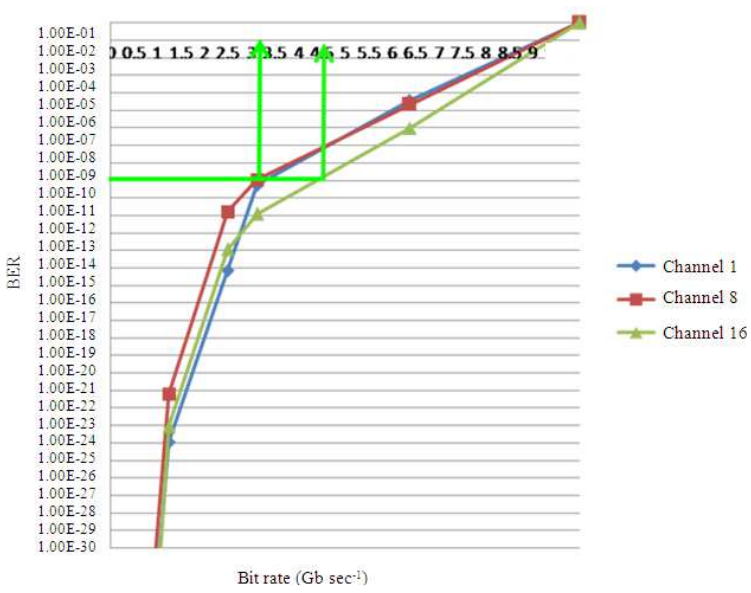

Fig. 4: BER Measured against Bit Rate over a $2.4 \mathrm{~km}$ distance under the condition of heavy rainfall
Figure 5 shows the effect of the different wavelength to the systems performance. The range for the wavelength is from 850-1550 nm based on commercial product in lightpointe and bit rate was set to $2.5 \mathrm{Gbps}$. The power transmitting was set to $10 \mathrm{dBm}$.

At the receiver, the 16-channels $2.5 \mathrm{Gbps}$ signals are shown in Fig. 6. Channel 1 has about $14 \mathrm{dBm}$ and channel 16 has about $4 \mathrm{dBm}$. The performance varies from channel to channel because different channel has different error floor. However, the BER of $10^{-9}$ is reachable.

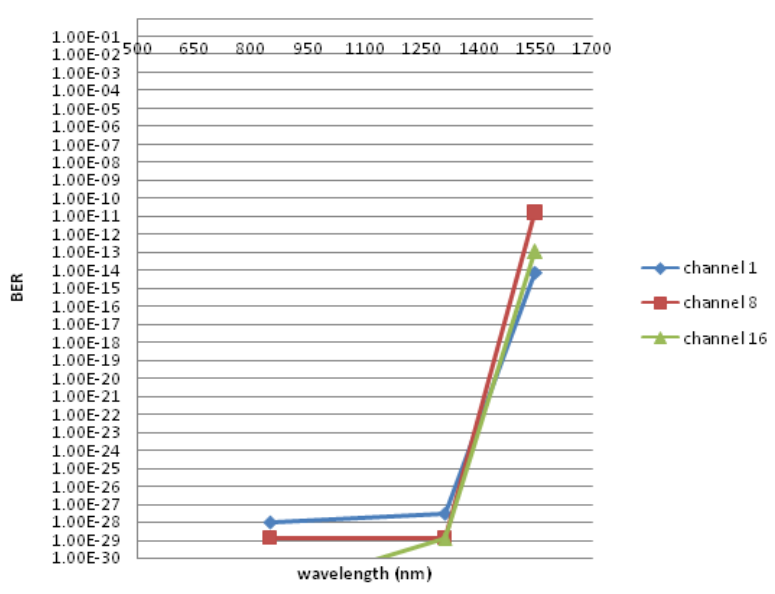

Fig. 5: BER in relation to increasing wavelength for WDM FSO system.

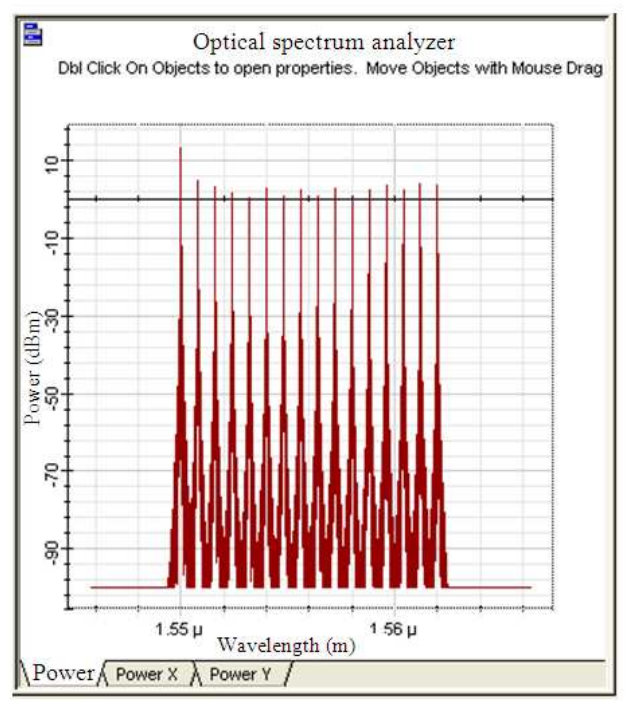

Fig. 6: Power of each channel 


\section{DISCUSSION}

From Fig. 3 shown above, BER appears to increase when the distance is increased because a longer distance will influence the performance of three of the measured means: loss, non-selective scattering and noise. From the graph, the highest distance that gives the best BER of $10^{-9}$ is at $2.4 \mathrm{~km}$ until $2.5 \mathrm{~km}$.

From the result in Fig. 4, increasing the bit rate will decrease the pulse width and affect the quality of the received signal. It is known to suffer dispersion under heavy rainfall and a loss in transmission. Changing the bit rate will also affect the noise associated with the receiver circuitry (Wayne, 2004). The result from the simulation shows that the BER for all channel almost the same where at BER of $10^{-9}$ the bit rate for 1,8 and channel 16 are 3.25-3.25 and 4.5 Gbps respectively. This is because the spacing between channels is sufficient to avoid crosstalk or interference.

From Fig. 5, the $850 \mathrm{~nm}$ wavelength produced a better result than $1550 \mathrm{~nm}$. With respect to transmission performance, availability and reliability of components, the 850 and $1550 \mathrm{~nm}$ wavelength ranges are the most economical and best performing wavelength ranges for operating FSO systems (LWPS, 2009). However $1550 \mathrm{~nm}$ wavelength is well suited for free space transmission due to its low attenuation, as well as the proliferation of highquality transmitter and detector components. Components include very high-speed semiconductor laser technology suitable for WDM operation as well as amplifiers (EDFA, SOA) used to boost transmission power. Because of the attenuation properties and component availability at this range, development of WDM free space optical systems is feasible.

\section{CONCLUSION}

From the results analysis, they show that the performance of WDM in FSO transmission is successfully achieved even though in worst case scenario. The conventional Intensity Modulation Direct Detection technique does not seem to be a promising candidate for long distance (more than $1 \mathrm{~km}$ ) in the same environment. So far, this technique, operating at $2.5 \mathrm{Gbps}$ bit rate, is presented over links of $0.8 \mathrm{~km}$ in heavy rainfall/low visibility (Lightpinte, 2010). However, WDM FSO system with the parameters specification as in the System Design section can be implemented in higher bandwidth and good performance. The capacity of WDM FSO is also possible to be increased up to 32-channels if any methods of error corrections or detection are introduced. Since the FSO transmission is totally depends on the weather condition and the obstacle between transmitter and receiver, the system only suitable for short distance and low bit rate.

\section{REFERENCES}

AC, 2011. Complete Wireless Solution for All Applications. Airlinx Communications, Inc.

Achour, M., 2002. Simulating atmospheric free-space optical propagation: Rainfall attenuation. Proceeding of the SPIE, Wed. 01 Aug., Optical Wireless Communications, Boston, USA., pp: 4635. DOI: $10.1117 / 12.464100$

Chan, V.W.S., 2006. Free-space optical communications. Light. Technol. J., 24: 47504762. DOI: $10.1109 /$ JLT.2006.885252

Cvijetic, N., D. Qian, J. Yu, Y.K. Huang and T. Wang, 2010. Polarization-multiplexed optical wireless transmission with coherent detection. J. Light. Technol., 28: 1218-1227.

Downing, J.N., 2004. Fiber Optic Communications. 1st Edn., Cengage Learning, Australia, ISBN: 1401866352, pp: 378.

Kim, I.I., R. Stieger, J.A. Koontz, C. Moursund and M. Barclay et al., 1998. Wireless optical transmission of fast ethernet, FDDI, ATM and ESCON protocol data using the terralink laser communication system. Opt. Eng., 37: 3143-3143. DOI: 10.1117/1.601981.

Kintaka, K., J. Nishii, S. Murata and S. Ura, 2010. Eight-channel WDM intraboard optical interconnect device by integration of add/drop multiplexers in thin-film waveguide. J. Light. Technol., 28: 1398-1403.

LWPS, 2009. Enterprise Campus Building-to-Building Connectivity and ROI Analysis of MillimeterWave (MMW) Radio Systems. LightPointe White Paper Series.

Ab-Rahman, M.S., H. Guna, M.H. Harun, S.D. Zan and K. Jumari, 2009. Cost-effective fabrication of self-made $1 \times 12$ polymer optical fiber-based optical splitters for automotive application. Am. J. Eng. Applied Sci., 2: 252-259. DOI: 10.3844/ajeassp.2009.252.259

Prokes, A., 2009. Atmospheric effects on availability of free space optics systems. Opt. Eng., 48: 066001066001. DOI: $10.1117 / 1.3155431$

Sinefeld, D. and D.M. Marom, 2010. Hybrid guidedwave/free-space optics photonic spectral processor based on LCoS phase only modulator. IEEE Photonics Technol. Lett., 7: 510-512.

Singh, J., M. Singh, S. Hassan, S.I. Ain and M. Fadzil, 2007. Rainfall attenuation and rainfall rate measurements in Malaysia comparison with prediction models. Am. J. Applied Sci., 4: 5-7.

Wayne, T., 2004. Electronic Communications Systems Fundamentals Through Advanced. 5th Edn., Prentice Hall, Upper Saddle River, ISBN 0130494925, pp: 1163. 